\title{
Testing miniaturized electrodes for impedance measurements within the $\beta$-dispersion - a practical approach
}

\author{
Uwe Pliquett ${ }^{1,3}$, Dieter Frense ${ }^{1}$, Markus Schönfeldt ${ }^{1}$, Christian Frätzer ${ }^{1}$, Yong Zhang ${ }^{1}$, Brian Cahill ${ }^{1}$, \\ Michael Metzen ${ }^{2}$, Andreas Barthel ${ }^{1}$, Thomas Nacke ${ }^{1}$ and Dieter Beckmann ${ }^{1}$ \\ 1. Institut für Bioprozess- und Analysenmesstechnik e.V., Heilbad Heiligenstadt, Germany \\ 2. Universität Bonn, Institut für Zoologie, Bonn, Germany \\ 3. E-mail any correspondence to: uwe.pliquett@iba-heiligenstadt.de
}

\begin{abstract}
Miniaturized electrodes are introduced in life sciences in a great number and variety. They are often designed for a special purpose without the need of quantitative analysis, such as for detecting cells or water droplets in a fluid channel. Other developments aim in monitoring a single quantity in a process where all other factors held constant.

To use miniaturized electrodes for quantitative measurements, their behavior should be known in detail and stable over time in order to allow a mathematical correction of the data measured.

Here we show test procedures for evaluating macroscopic but also microscopic electrodes. The most important quality parameters for electrode systems used in life science are the electrode impedance, its stability, the useful frequency range as well as the limits for applied stimulus without driving the electrode system into a non-linear region of the current/voltage relation. Proper electrode design allows a bandwidth from $100 \mathrm{~Hz}$ up to some $\mathrm{MHz}$ for impedances ranging over decades from $50 \Omega$ up to several $\mathrm{M} \Omega$.
\end{abstract}

Keywords: Impedance, electrode polarization, voltammetry, miniaturization

\section{Introduction}

The vision is clear and often pronounced. Microelectrodes open up new horizons for bio-impedance measurements [17]. This raises questions: How much will microelectrodes solve old problems? But more important is to ask: What problems need to be solved for optimal usage of microelectrodes $[8 ; 9]$ ?

As widely accepted, the measurement of passive electrical properties of cells and cell suspensions is a simple, fast and non invasive method [10-12]. Since electrical cell behavior is often well correlated with quality measures, impedance measurement is a good candidate for quality assessment for instance in medicine [1;13-15], food industry [16-19] or biotechnology [20;21].

Besides well established arrangements with electrode dimension of centimeters, a trend to miniaturized electrode systems is obvious. The reason is the development of single cell monitoring but also the usage of miniaturized impedimetric sensors for minimal sample volume [2;4;22-24].
It was up to the 60ies of last century not simple to make miniaturized electrodes. Today, almost any electrode shape and size even down to molecular level are feasible [25;26]. Based on theoretical prediction, like FEM (finite element method), most researchers are today sure about the behavior of the electrode systems in use [27]. Problems are not always obvious. For instance, the simulation of the field distribution at equally shaped electrodes but with different size yields the same pictures. But calculating the geometry factor $d / A$ (distance / surface area) for a simple electrode geometry reveals marked differences. For instance, the geometry factor $\mathrm{k}$ for two plan parallel electrodes of $A=1 \mathrm{~cm}^{2}$ and $d=1 \mathrm{~cm}$ is $k=d / A=1 \mathrm{~cm}^{-1}$. The same proportion of the electrode geometry but with $A=(10 \times 10) \mathrm{cm}^{2}$ and $d=10 \mathrm{~cm}$ will yield $k=0.1 \mathrm{~cm}^{-1}$. If we change direction towards very small electrodes, we find theoretically only a change of the geometry factor. Does this mean that approaching small dimension will only change a constant factor?

The experiment reveals: Microelectrodes are not simply small macroelectrodes [28]!

Here we present the characterization of a well defined macroscopic electrode system and show how to interpret the impedance behavior of miniaturized electrodes.

The paper does not contain sophisticated science but it is a rather practical approach for those researchers employing bio-impedance measurement as tool without having strong background in electrochemistry. It should help to make features found in experiments with miniaturized electrodes more understandable on a way for quantitative interpretation of data.

\section{Impedance of cells}

A very simple approach for modeling cells is an electrolytic solution (cell plasma) surrounded by an insulating membrane (plasma membrane). The extracellular space is filled with electrolyte as well, but may have a very distinct conductivity with respect to the conductivity of the cytoplasm. Although the cytoplasm contains membrane systems (e.g. endoplasmatic reticulum, Golgi apparatus, nuclear mem- 
brane or other membrane enclosed organelles) a pure resistive model for this part is often sufficient for a practical approach $[29 ; 30]$.

Since intact cell membranes show a low conductance, the low frequency current ( $\mathrm{f}<10 \mathrm{kHz}$ ) will predominantly flow around the cells [31-33]. Only at higher frequencies (f $>1 \mathrm{MHz}$ ), when the membranes are electrically shortened, the impedance decreases due to the accessibility of the cytoplasm as current path which yields the $\beta$-dispersion. The impedance at low frequencies gives information about the cell volume fraction since isolating cell membranes decrease the current path. This however, needs either calibration with known cell densities or the knowledge of the conductivity of the extracellular electrolytes as well as the electrode geometry. More advanced is the impedance measurement over the entire $\beta$-dispersion and extracting the cell volume fraction using suitable physical models [34]. Since only cells with intact, i.e. insulating, membranes contribute to the $\beta$-dispersion, an important quality parameter for tissue and cell suspension related to the fraction of damaged cells can be derived from the impedance spectrum $[1 ; 3 ; 35$ 39].

The influence of the cells on the measured signal will vanish at cell volume fraction below about $0.1 \%$, usually already earlier [1]. This means, for large cells $\left(\mathrm{r}_{\text {cell }}>10 \mu \mathrm{m}\right)$, a detection limit of around $2 \cdot 10^{5}$ cell $/ \mathrm{mL}$ is reasonable while for detecting small bacteria almost $10^{9}$ cells $/ \mathrm{mL}$ are necessary.

However, ionic cell metabolites can change the conductivity of the suspension medium, especially if media with low conductivity are used. In this case, the volume fraction of the cells excluded from the volume carrying the current is unimportant. Only the concentration of the ionic metabolites in the extracellular electrolyte matters. The release of ionic species from the cells is either governed by the normal metabolism or due to cell lysis either by osmotic shock [40;41] when suspended in distilled water or by treatment like heat shock, sonication or electroporation $[16 ; 17 ; 42]$.

At frequencies in the microwave region (e.g. $f=2.54 \mathrm{GHz}$ ), a specific absorption of water molecules allow the assessment of water content. Since the water content changes with aging of tissue, e.g. agricultural products, it is related to quality measures. Moreover, it was shown to be sensitive to the dry organic mass in slurries like biogas substrate [43].

\section{Measurement methods}

Besides well established methods for low frequency impedance measurements like bridge balancing, the application of a voltage to the MUT (material under test) and monitoring the total current (potentiostatic measurement) or vice versa, controlling the current while monitoring the voltage dropping at the MUT (galvanostatic measurement) is widely used [44]. For objects with linear U/I-characteristic (voltage/current) it is unimportant whether the measurement is done in time or frequency domain.

For dielectric spectroscopy in a frequency range above $300 \mathrm{MHz}$ parasitic elements will prevent accurate measurements by simply measuring current and voltage. In order to have a defined connection to the MUT, transmission lines with well known wave impedance are used together with network analyzers. Network analyzers monitor not only the energy transmitted by the MUT but also the reflected wave [45;46].

Many applications of bioimpedance measurement make use of galvanically coupled electrodes. The common problem with these electrodes is the electrode polarization in contact with aqueous electrolytes [28;47]. However, even if galvanically decoupled electrodes, e.g. metal electrodes passivated by a layer of dielectric material, are used, the measured results often depart from the prediction.

\section{Electrode impedance}

The most important frequency range for characterization of cells, tissue, slurries or for biosensors is between dc and 10 $\mathrm{MHz}$ which includes the conductivity of suspension media but also the $\beta$-dispersion arising from the polarization of intact cell membranes [48] and the microwave range (2-4 GHz) where the water content predominantly influences the impedance [49]. While antennas and galvanically decoupled electrode systems dominate in the microwave range, galvanically coupled electrodes are preferred for the low frequency range.

When a metal electrode touches an aqueous electrolyte, metal ions go into solution building up a charged layer at the surface [50-52]. Together with counterions a rigid double layer at the electrode, the so called Helmholtz layer (Fig.1) is formed. Weaker electrostatic forces from the charged metal surface are responsible for the creation of a diffuse layer. The mobility of ions in this Gouy-Chapman layer is limited by diffusion.

The impedance of the interface between the electrode and electrolyte arises from the charge transfer within the Helmholtz layer and the impedance of the Gouy-Chapman layer $[45 ; 46 ; 49 ; 53]$. Two resistive elements can be distinguished; the almost ohmic Nernst impedance governed by the charge transfer $\left(\mathrm{R}_{\mathrm{c}}\right)$ and the Warburg impedance $\left(\mathrm{Z}_{\mathrm{W}}\right)$ arising from the diffusion limitation within the GouyChapman layer (Fig.2).

Other then the Nernst impedance, the Warburg impedance is frequency dependent due to the diffusion process of the charge carriers. It can be modeled using a constant phase element (CPE). Because the charge mobility within the electrical double layer is limited, it behaves like a lossy capacitor $\left(\mathrm{C}_{\mathrm{dl}}\right)$. Independent of the electrode behavior is the resistance of the bulk electrolytes $\mathrm{R}_{\text {bulk }}$. 


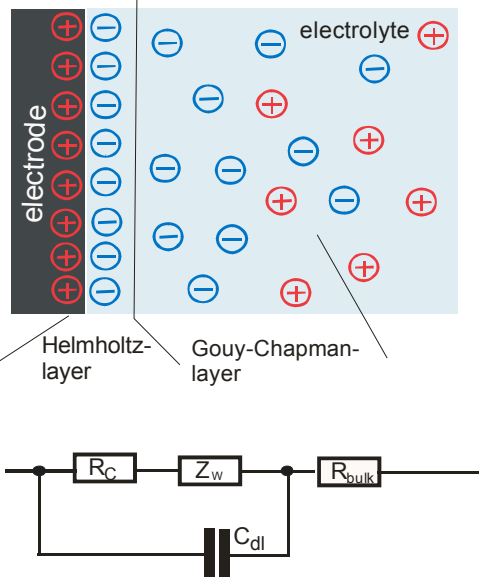

Fig.1: Interfacial layers between metal contact and electrolyte

The potential within the Helmholtz layer changes linearly. Due to the thickness on the order of $0.2 \mathrm{~nm}$, even potential differences less than a volt yield a field strength of more than $10^{9} \mathrm{~V} / \mathrm{m}$. Due to the vanishing electrostatic forces in the Gouy-Chapman layer, an exponential decay is found in this region. Thus, the thickness of this layer is defined by the Debye screening length $\lambda_{\mathrm{D}}$, which can be calculated by:

$$
\lambda_{D}=\sqrt{\frac{2 N_{A} e^{2} I}{\varepsilon_{r} \varepsilon_{0} k_{B} T}}
$$

with the ionic strength

$$
I=0.5 \sum_{i} z_{i}^{2} c_{i}
$$

$N_{A}$ is Avogadro's constant $\left(6.023 \cdot 10^{23} \mathrm{~mol}^{-1}\right), e$ is the elementary charge $\left(e=1.602 \cdot 10^{-19} \mathrm{C}\right), \varepsilon_{0}$ is the permittivity of vacuum $\left(\varepsilon_{0}=8.85 \cdot 10^{-12} \mathrm{As} / \mathrm{Vm}\right)$ and $k_{b}$ is Boltzman's constant $\left(k_{b}=1.38 \cdot 10^{-23} \mathrm{~J} / \mathrm{K}\right) . T$ is the absolute temperature and $\varepsilon_{\mathrm{r}}$ is the relative permittivity. The number to use for $\varepsilon_{\mathrm{r}}$, where mostly $\varepsilon_{\mathrm{r}}=78$ is assumed at room temperature for aqueous electrolytes, is not always clear since the relative permittivity in a region of hindered mobility decreases. Note, that the concentration is usually given in $\mathrm{mol} / \mathrm{L}$ which is off by a factor of 1000 with regard to the basic SI units and needs to taken into account for calculation of $\lambda_{\mathrm{D}} . \mathrm{z}$ is the charge carried by the $\mathrm{i}$-th specimen and $\mathrm{c}$ the concentration.

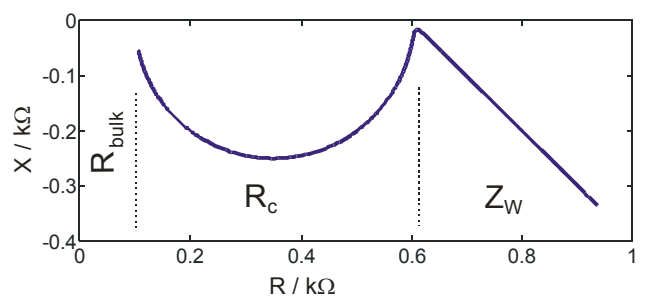

Fig.2: Impedance of a hypothetical metal electrode $\left(A=1 \mathrm{~mm}^{2}\right)$ in contact with $1 \mathrm{mM} \mathrm{KCl}\left(R_{\text {bulk }}=100 \Omega, R_{c}=500 \Omega, C_{d l}=30\right.$ $\mathrm{mF} / \mathrm{cm}^{2}, D=5 \cdot 10^{-8} \mathrm{~m}^{2} / \mathrm{s}, T=296.15 \mathrm{~K}$ ) in a frequency range from $100 \mathrm{~Hz}<f<100 \mathrm{MHz}$ with undisturbed diffusion layer
The extent of the electrical double layer is negligible, if macroscopic electrodes with electrode spacing on the order of millimeters or centimeters are used [54]. However, it may be important if one tries to detect internal structures electrically using submicrometer electrodes or a conductive AFM-tip (atomic force microscopy, special tips). As seen in Fig.3, the double layer thickness is less than a nanometer for electrolytes with physiological salt concentration $(140 \mathrm{mM})$, but can reach more than a micrometer for distilled water $(0.1 \mu \mathrm{M})$.

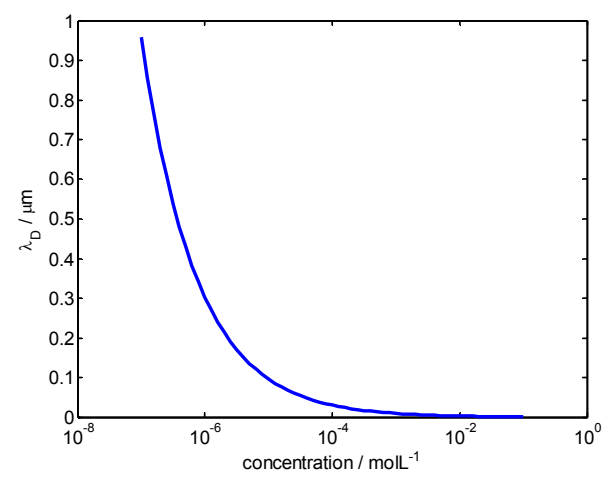

Fig.3: Debye screening length depending on the concentration of a 1:1 electrolyte. Note, the symbol $\lambda_{\mathrm{D}}$ was chosen rather than $\kappa$ as found in literature in order not to disturb with the conductivity where $\kappa$ is used as well.

The electrode impedance varies greatly with respect to the electrode potential as seen by cyclic voltammetry (Fig.4) [55]. Theoretically, the entire electrode impedance can be calculated [56]. However, as seen in Fig.4 even for electrodes of the same material and identical geometry, the current/voltage relation differs making the impedance dependent on the electrode potential. The electrode current $I=A J_{0}$ as function of the electrode potential $E$ (measured against the $\mathrm{H}^{+}$-electrode) is governed by the Butler-Volmer equation. $A$ is the electrode surface and $J_{0}$ the exchange current density.

$$
I=A J_{0}\left(e^{\frac{(1-\alpha) n F}{R T}\left(E-E_{e q}\right)}-e^{\frac{-\alpha n F}{R T}\left(E-E_{e q}\right)}\right)
$$

This equation assumes both, anodic as well as cathodic reactions at the electrode where $E_{e q}$ is the electrode potential when the total current is zero, i.e. the electrochemical equilibrium is reached. $n$ is the molar fraction, $F$ is the Faraday constant $\left(F=e N_{A}=9.610^{4} \mathrm{C} / \mathrm{mol}\right)$ and $R$ the universal gas constant $\left(R=k_{b} N_{A}=8.3 \mathrm{~J} / \mathrm{K}\right) . \quad \alpha$ is a dimensionless symmetry factor and $T$ the absolute temperature. The charge transfer resistance is therefore $R_{c}=E / T$. It becomes infinite, when $E=E_{e q}$, i.e. no chemical reaction takes place. 


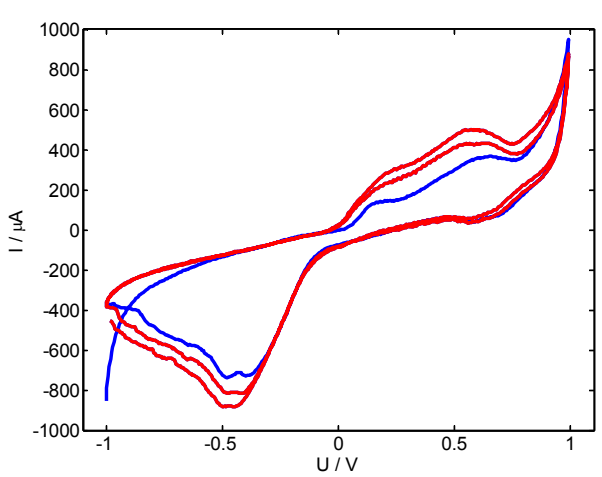

Fig.4: Cyclic voltammogram of two different gold electrodes in $140 \mathrm{mM}$ PBS (phosphate buffered saline). The electrode area was $2 \mathrm{~cm}^{2}$ and the voltage slope was set to $50 \mathrm{mV} / \mathrm{s}$.

Although the Butler-Volmer equation looks difficult, it is rather simple to interpret. $A J_{0}, E_{e q}$ and $E$ are measures got from voltammetry experiments, in ideal case with zero ramp slope. Moreover, if the electrolytic system is known and well defined, $E_{e q}$ is known as well. If the ramp slope is too high, the kinetics of the chemical reaction will influence the U/I-characteristic because the time for reaching a steady state is not sufficient. $n, F, R, T$ are constant or easy to control. It should be noted, that $n F$ is the number of charge carriers and $n F\left(E-E_{e q}\right)$ is the electrical energy while $R T$ is the thermal energy. The only somehow arbitrary parameter is $\alpha$. It can be determined from the $U / I$-slope in voltammetry experiments. The both exponentials (modified Boltzman-factors) yield the increase $\left(\mathrm{e}^{(1-\mathrm{x})}\right.$, anodic reaction) and decrease ( $\mathrm{e}^{-\mathrm{x}}$, cathodic reaction) of current arising from species with different polarity.

The infinite-length-Warburg-impedance [51] is a constant phase element with a phase of $45^{\circ}$ for undisturbed diffusion layers. This means the real and the imaginary part is always the same.

$$
Z_{W}(j \omega)=\frac{A_{W}}{\sqrt{\omega}}(1-j)
$$

$A_{W}$ is the Warburg element:

$$
A_{W}=\frac{R T}{z^{2} F^{2} A c \sqrt{2 D}} .
$$

$\omega$ is the angular frequency and $j$ is the square root of -1 . With the conductivity $\kappa, D$ can be estimated for aqueous 1:1 electrolytes by

$$
D=\frac{R T \kappa}{c}
$$

A charged surface of the electrode will influence the impedance as well but also the electrode potential especially for small potential differences between the electrode and the electrolyte. The surface potential is the $\zeta$-potential which equals the voltage drop across the Gouy-Chapman layer [55]. Since this layer is built up by weak electrostatic forces, a flow of the medium contacting the electrode will disturb this layer. The Gouy-Chapman layer vanishes at sufficiently high speed of the medium contacting the electrode. This has two consequences - a lower electrode impedance due to the loss of the Warburg impedance and the potential of the electrolyte equals the $\zeta$-potential. Moreover, the phase angle of the Warburg impedance deviates from $45^{\circ}$. It is unimportant for electrodes contacting unstirred solution but needs to be taken into account in fluidic systems or when the medium is stirred, e.g. when cell suspensions are measured and stirring is applied for preventing settling of the cells.

It should be noted, that analytic equations presented are only valid for well defined boundary condition. Thus, they often provide only raw estimations. However, the theoretical prediction coincides well with the measurement for defined geometries of macroscopic electrodes and even more important for defined electrolytes [8]. In bioimpedance measurements specific properties of the electrode arrangement are often not known. Calibration procedures and the design of sophisticated electrode systems like tetrapolar electrodes can be used for compensation of the electrode behavior. But, not everything is always applicable.

\section{Bipolar and tetrapolar electrodes}

The simplest electrode configuration is the bipolar electrode. The calibration is in general done by using electrolytes with defined behavior such as conductivity and charge mobility [54]. $\mathrm{KCl}$ is a strong electrolyte with almost equal mobility of the $\mathrm{K}^{+}$and the $\mathrm{Cl}^{-}$- ions and dissociates up to a concentration of $3 \mathrm{M}$ almost completely. The conductivity over a wide range of temperatures and concentrations is listed in text books. The geometry constant $k$ of any electrode in the low frequency region can be found by filling the measurement cell with $\mathrm{KCl}$ of known concentration and temperature measuring the real part $G$ of the admittance. From the relation between the conductivity and conductance follows $k=\kappa_{C} / G$. Using the calibrated chamber implies that the electrode behavior must not change during the measurement procedure. But, according to the considerations for the electrode impedance, exchanging the electrolyte influences the electrode impedance as well. Since this impedance is in series to the MUT, it is almost impossible to account for this. In this case, the electrode impedance should be as low as possible, for instance by using big electrodes or electrodes with a fractal surface $[57 ; 58]$. Well established electrodes for this purpose are platinum black or iridium sputtered electrodes as they are used for pacemakers. In recent years, especially due to new developments in nanotechnology a great variety of fractal surfaces was presented.

A more elegant way for minimizing the influence of electrode polarization is the use of separate electrodes for current application and voltage measurement (tetrapolar interface) [49]. In this case an outer pair of electrodes is used for current injection while the voltage is monitored at separated inner pair of electrodes. Any voltage drop at the 
outer electrodes will not be monitored at the inner electrodes. If the voltage difference at the inner electrodes is monitored by a high impedance difference amplifier, the electrode potential, which should be the same at both inner electrodes, will not influence the measured potential difference.

For high frequency application, electrode polarization is mostly negligible due to the capacitive behavior of the electrical double layer. Moreover, the higher parasitic capacitances decrease the accuracy of tetrapolar interfaces, which makes bipolar interfaces the better choice for high frequency measurements.

When miniaturized electrodes are used, in many applications only bipolar interfaces, like interdigitated electrodes are considered. The suitability of tetrapolar electrodes is not only limited by the higher space requirement but also by the need to handle four leads instead of two. Thus, in MEMS (micro electro mechanical systems) predominantly bipolar electrodes are found.

\section{Confidence region of electrodes}

If only a distinction between two very different liquids like oil and water is needed, a single frequency measurement using a bipolar electrode will be sufficient. However, when a quantitative result is required, a careful test of the confidence region with respect to ionic strength and frequency range should be known. Especially miniaturized electrodes show often considerable deviation from ideal behavior because of the increasing influence of parasitic elements. An example of how complicate the electrical modeling of just a simple looking interdigitated electrode passivated with silicon nitride can be, is shown in Fig.5. Fortunately, not all parasitic elements dominate the impedance measurement. However, this needs to be tested for each individual electrode system and application.

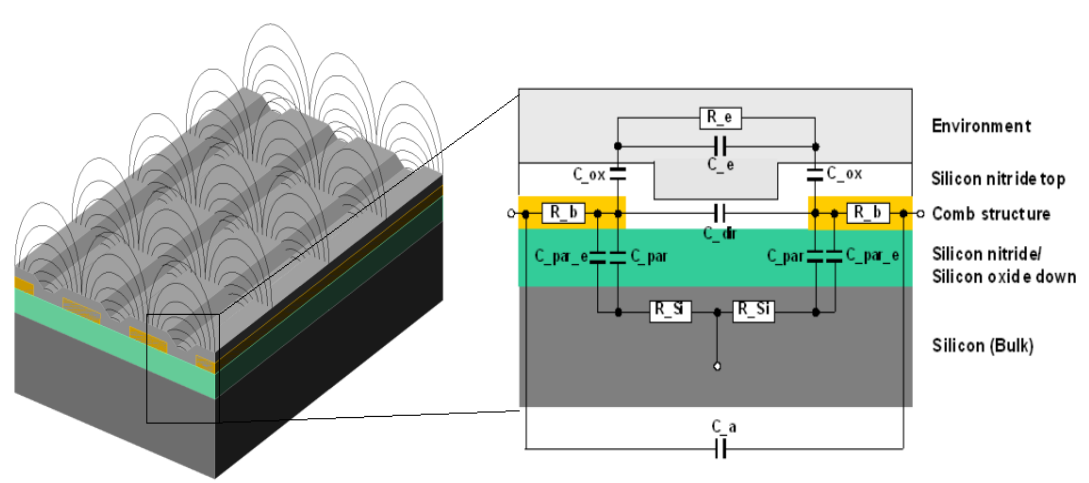

Fig.6: Tetrapolar interface consisting of four stainless steel rings with a diameter of $6 \mathrm{~mm}$. The electrode system is molded in PMMA (poly-methylene-methacrylate).

Another electrode configuration under test was a gold electrode made from a rewritable CD (Fig.7). Preparing electrodes from $\mathrm{CDs}$ is a cheap way towards high quality gold electrodes [60]. High performance CD-Rs are composed of polycarbonate with a 24-karat gold layer of $30 \mathrm{~nm}$. This is covered by a transparent lack. After removing the lack, a photoresist is used for masking the etching step, where the gold is removed by iodide etchant. The illumination of the photoresist using UV-light is masked by transparencies printed on an ordinary LASER-printer in high quality mode. Further processing involves the passivation of the leads to the active area of the electrodes. Structures down to $30 \mu \mathrm{m}$ were made, mostly for use in microfluidic channels formed by PDMS (silicone rubber, poly-dimethylsiloxan)

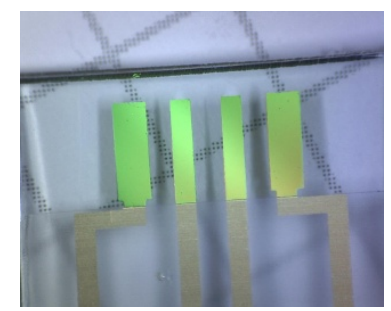

Fig.7: Tetrapolar electrode made from a CD-R. The dimension of the inner electrodes is $100 \mu \mathrm{m}$. For electrical characterization of the electrodes, measurements in bulk electrolytes were done with the leads passivated using Tesa tape (Bayersdorf AG, Hamburg, Germany).

distances between $1 \mu \mathrm{m}$ and $150 \mu \mathrm{m}$ are built on a silicon wafe with silicon nitride as passivation layer. Sensors like these are useful as galvanically decoupled impedance sensors. (CiS Forschungs-institut für Mikroelektronik und Photovoltaik GmbH, Erfurt, Germany)

It is convincing to test the range where electrodes yield predictable results by employing natural laws like
For testing the electrical behavior of the electrodes with different dimension four single electrode patches with $0.125 \mathrm{~mm}^{2}, 1 \mathrm{~mm}^{2}, 4 \mathrm{~mm}^{2}$, and $16 \mathrm{~mm}^{2}$ were prepared. We used a monopolar approach with a large, iridium sputtered 
counter electrode for impedance measurements and a platinum electrode together with a silver/silver chloride electrode $(\mathrm{Ag} / \mathrm{AgCl})$ immersed in $3 \mathrm{KCl}$ (potassium chloride) as reference electrode for cyclic voltammetry experiments.

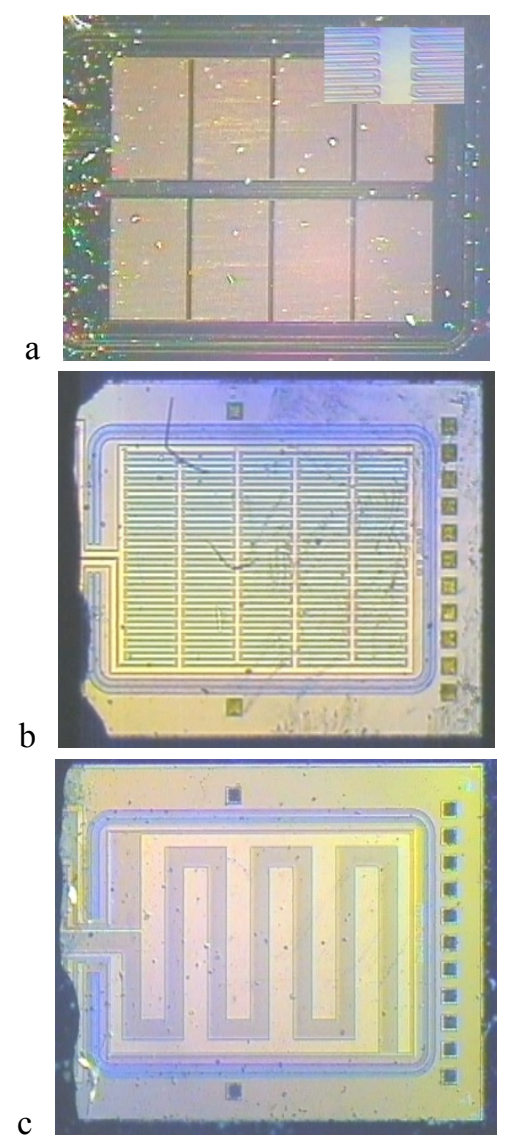

Fig.8: Interdigitated electrodes with different structural dimension a) $3 \mu \mathrm{m}$ (enlarged vie in insert image), b) $20 \mu \mathrm{m}$ and c) $150 \mu \mathrm{m}$, developed by $\mathrm{CiS}$ Forschungsinstitut für Mikroelektronik und Photovoltaik GmbH, Erfurt, Germany. These electrodes are arranged in blocks for the small electrode distance (up to $50 \mu \mathrm{m}$ ) while larger electrodes are made of one block (Fig.8c).

Especially for the use in microfluidic devices, galvanically decoupled electrodes were developed (Fig.8). The electrodes are interdigitated bipolar systems manufactured in a CMOS process and passivated by silicon nitride ( $\mathrm{SiN})$. The electrode devices were mounted on PCB-material and the bonds are molded in polyurethane.

\section{Impedance measurement}

Different impedance measurements systems such as Solartron 1260 (Schlumberger, UK), HP 4194 A (Agilent, USA) or IMPSPEC (MEODAT, Germany) were used with custom made front ends, allowing measurements of impedances from $\Omega$ up to G $\Omega$ in a frequency range from $\mathrm{mHz}$ up to $200 \mathrm{MHz}$ depending on the impedance of the MUT. Front-ends for potentiostatic measurements were available as well as a symmetric, offset free current source for galvanostatic excitation [56]. If not otherwise noted, the temperature was $25^{\circ} \mathrm{C}$.
Impedance measurements in the microwave region are behind the scope of this paper.

\section{Cyclic voltammetry}

A potentiostat/galvanostat (Model 2059, AMEL instruments, Italy) was custom programmed using a PC equipped with an analog interface card. In all voltammetry experiments a platinum sheet was used as counter electrode while an $\mathrm{Ag} / \mathrm{AgCl}$-electrode immersed in $3 \mathrm{M} \mathrm{KCl}$ served as reference. Besides experiments for checking and calibrating the system where potassium-hexacyanoferrate electrolyte was used, all experiments shown here were done with biological relevant solutions like $\mathrm{KCl}$ or $\mathrm{PBS}$.

\section{Electrical stability}

The potential difference for the electrode under test was measured against an $\mathrm{Ag} / \mathrm{AgCl}$ - electrode using an electrometer amplifier with an input impedance of $50 \mathrm{~T} \Omega$. A measurement was done every $30 \mathrm{~s}$ for several hours up to days. Especially for assessing the stability of surface modified sensor electrodes, stirring with different speed was introduced as additional independent parameter. Stirring influences the Gouy-Chapman layer and yields an effective electrode potential which depends on the $\zeta$-potential [61]. Moreover, due to shear forces surface modified electrodes can degrade faster in a stirred environment or in microfluidic channels.

Another critical aspect is the water uptake of materials used for packaging or supporting the electrode system. Unfortunately, many materials, like most PCB-materials and polymers take up enough water in order to change significantly the parasitic elements of the electrode system.

Bio-fouling was assessed for several electrode systems but is too complex to be presented here.

\section{Electrode inspection}

Several of the tested electrode devices showed a fast degradation. This was most pronounced for $\mathrm{Ag} / \mathrm{AgCl}$-electrodes prepared on gold surfaces. SEM (scanning electron microscopy) was employed for visualizing defects at the surface responsible for accelerated electrode corrosion. Using EDX (energy dispersive X-ray spectroscopy) even the origin of surface defect could be identified. For routine assessment of electrode surfaces, ordinary light microscopy was used.

\section{Characterization of electrodes - procedures and results}

Electrode development focuses in general on the electrode material and the geometry. The material should be chemically inert and mechanically stable and the geometry should be optimized for the object [60]. Despite much work is done in this field, even in our days poor electrode construction can be found. Moreover, measuring with non-suited electrode systems results in low reproducibility of the mea- 
surement and is often a reason for rejecting impedance measurement as method.

Practical tests of electrode systems for bioimpedance measurements should involve impedance spectroscopy with known substances over a range of ionic strength, cyclic voltammetry of the single electrodes as well as for reversible electrodes (e.g. $\mathrm{Ag} / \mathrm{AgCl}$ ) stability tests for the electrode potential. This characterizes an electrode system with respect to the useful frequency range, the maximum voltage to be applied as stimulus, the conductivity range of the MUT and the stability over time.

\section{Impedance spectroscopy and calibration}

When measuring in a frequency range between $10 \mathrm{~Hz}$ and $10 \mathrm{MHz}$, the influence of the electrode system changing with frequency is often not considered. This is well supported, since resonance points are only expected above this frequency range and transmission line behavior is negligible. This means, one would expect to calibrate the electrode with an electrolyte of known conductivity (e.g. $100 \mathrm{mM}$ $\mathrm{KCl}$ measured for instance at $400 \mathrm{~Hz}$ ) for the entire frequency range. The geometry factor will be expected to be the same for all other concentrations or even electrolytes as well. An example, that this is only partially true is given in Fig.9 using an electrode system as shown in Fig.6.

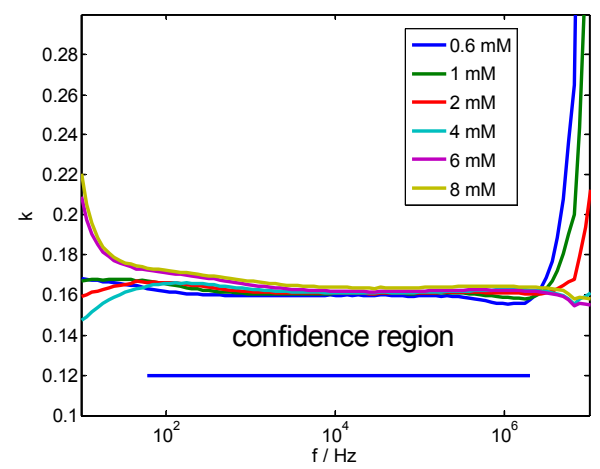

Fig.9: Geometry coefficient calculated over the frequency range for an electrode shown in Fig.6.

The electrodes were polished and impedance measurements in a frequency range between $10 \mathrm{~Hz}$ and $10 \mathrm{MHz}$ were done at dilution series of $\mathrm{KCl}$ at $25^{\circ} \mathrm{C}$. $\mathrm{KCl}$ was chosen for the equal mobility of cations and anions which prevents the buildup of diffusion potentials.

The geometry coefficient should be theoretically constant. However, as seen in Fig.9, slight departure from ideal behavior occurs. Besides some uncertainty of the $\mathrm{KCl}$ conductivity (measured with LF-300, WTW Weinheim, Germany) parasitic elements of the electrode construction cause a systematic error. This can be compensated using a physical model of the electrode, which is behind the scope of this paper. The confidence region with respect to the frequency range can be derived from measurements as shown in Fig.9. However, it is still somehow arbitrary, since there does not exist any normalized criteria.

\section{Kohlrausch's Law}

The most convincing check of an electrode system is the validation by a natural law. Strong electrolytes show a decrease in molar conductivity with increasing concentration due to the screening effect between ions. Kohlrausch found empirically the square dependence of the molar conductivity $\Lambda_{\mathrm{m}}$ on the square root of the concentration.

$$
\Lambda_{m}=\Lambda_{0}-\alpha \sqrt{c} \text { with } \Lambda_{\mathrm{m}}=\kappa / \mathrm{c}
$$

$\Lambda_{0}$ is the limiting molar conductivity at infinite dilution, i.e. the molar conductivity under condition without any screening of ions. Kohlrausch's law yields a straight line for the confidence region of $k$ (Fig.10).

\section{Stability of the electrode potential}

Since impedance measurements are done with ac, any instability in the dc-electrode potential should not influence the measurement result. However, when measuring at low frequency, e.g. below $10 \mathrm{~Hz}$, an unstable dc potential can yield unpredictable results. The potential stabilizes with moderate current through the electrode. Thus, it is less important for bipolar interfaces but a great problem for tetrapolar electrode systems, because of the high ohmic coupling of the voltage monitor.

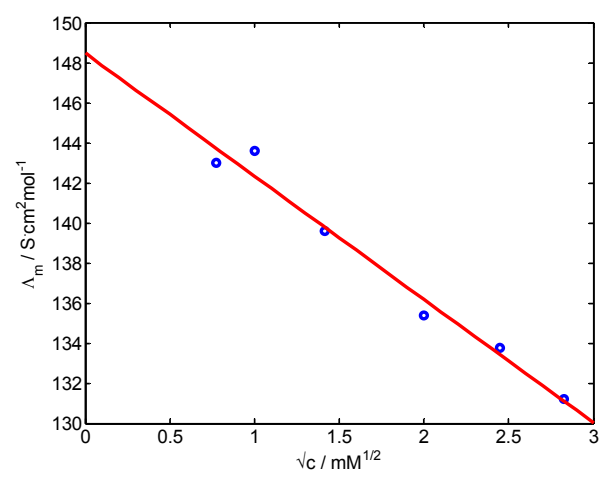

Fig.10: Molar conductivity of $\mathrm{KCl}$ with varying concentration. The conductivity in the frequency range of the confidence region was corrected by the conductivity of pure water $\left(\kappa_{w}=6.41 \mu \mathrm{s} / \mathrm{m}\right.$ at $\left.25^{\circ} \mathrm{C}\right)$.

The electrode potential of metal in aqueous electrolytes is governed by the metal ions in electrochemical equilibrium with the metal. Because of the very low activity of the metal ions even small changes in their total number will change the electrode potential on the order of several millivolt. Especially for monitoring or as reference reversible electrodes are used. The common choice for measurements at biological MUT is the $\mathrm{Ag} / \mathrm{AgCl}$ electrode. Metallic silver is covered by the hardly soluble silver chloride. The electrode potential is determined by the equilibrium constant $\mathrm{K}_{\mathrm{D}}$ of the silver chloride dissociating into silver and chloride ions which is only dependent on the chloride ion concentration of the electrolyte. In usual practice an $\mathrm{Ag} / \mathrm{AgCl}$-electrode is immersed into $3 \mathrm{M} \mathrm{KCl}$ which is a 1:1 electrolyte with almost equal ionic mobility. Together 
with the high ionic strength only negligible diffusion potential appears. In miniaturized electrode systems it is often easier to expose the silver chloride directly to the MUT. Since the chloride activity in biological systems (e.g. suspension medium) is either known or changes only negligibly, even bare $\mathrm{Ag} / \mathrm{AgCl}$ electrodes show a stable electrode potential. In applications like voltage monitoring across a pair of electrodes with equal properties, the actual electrode potential is even not important.

For this purpose gold electrodes made from CD-Rs were cathodically plated for $20 \mathrm{~min}$ with silver (electrolyte silver, Conrad, Germany) and subsequently for $10 \mathrm{~min}$ anodically chloridized in $1 \mathrm{M} \mathrm{HCl}$. Silver wire was used as counter electrode. The current density was set to $1 \mathrm{~mA} / \mathrm{cm}^{2}$.

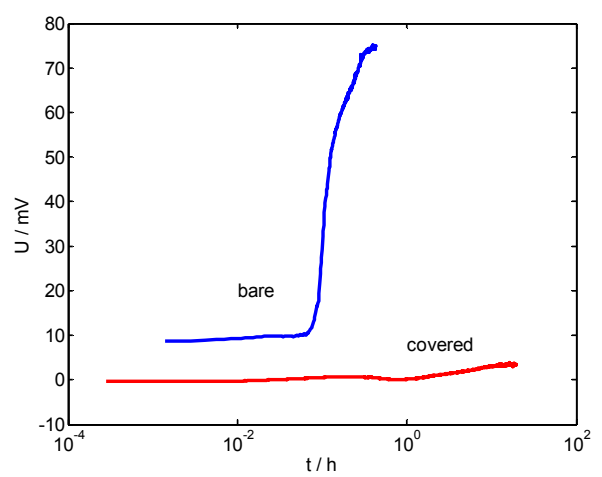

Fig.11: Potential of surface modified electrodes with respect to an $\mathrm{Ag} / \mathrm{AgCl}$-electrode immersed in $3 \mathrm{M} \mathrm{KCl}$. The chamber was filled with $140 \mathrm{mM}$ PBS. A magnetic stirrer was used with a speed of about $500 \mathrm{rpm}$. Both test electrodes were made by plating silver onto gold with subsequent anodic oxidation in $\mathrm{HCl}$. While one electrode was left bare, the other one was covered using a polyurethane gel.

When assessing the stability sometimes even without any electrical stimulus the electrode potential was changing by progressing corrosion in electrolytes like PBS or $\mathrm{KCl}$. Examination using SEM (scanning electron microscopy) and EDX (energy dispersive X-ray spectroscopy) showed microscopic defects at the surface, where gold and silver were both exposed to the electrolyte (Fig.12).

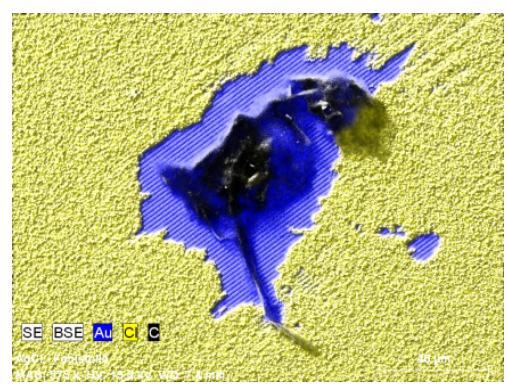

Fig.12: Surface defect at a $\mathrm{Ag} / \mathrm{AgCl}$ covered gold electrode. The electrode was made from a CD-R carrying a 24-karat gold layer. The surface was scanned using EDX (energy-dispersive X-ray spectroscopy). The rough surface is $\mathrm{AgCl}$ while the pure gold is found around the impurity which consist of organic dust. The stripe structure of the gold is a feature of the CD. (Image by Holger Rothe, iba Heiligenstadt)
The origin of these defects is not clear just from microscopic inspection. However, using EDX, we found organic dust as reason for non-uniformly covered electrodes (Fig.12). The lines seen in the gold-region are due to the structure of the CD-R (compact disk), the material used for making the electrode.

In conclusion, we prepared our electrodes under a flow hood as clean as possible and used dried air jet for cleaning the electrodes from dust particles. This resulted in electrode surfaces to be stable (within $2 \mathrm{mV}$ over more than a day). Quite different behaviour was found when the electrolyte was stirred. The electrode potential changes by more than $60 \mathrm{mV}$ after about $30 \mathrm{~min}$, depending on the speed of stirring $(10-500 \mathrm{rpm})$. Inspection of the electrode showed a complete dissolved AgCl-layer. This is understandable because silver chloride is hardly soluble but NOT insoluble. Stirring accelerates the dissolution due to overcoming the diffusion barrier. In order to prevent the stirring at the surface, we covered the electrode with a several micrometer thick layer of polyurethane gel (water resistive wood glue). This gel becomes hydrated and shows a similar conductivity as the surrounding electrolyte. The electrodes appeared to be stable, at least for a day (Fig.11).

\section{From macroscopic towards microscopic electrode dimensions}

In order to assess the impedance of electrodes with dimensions ranging from $16 \mathrm{~mm}^{2}$ down to $0.125 \mathrm{~mm}^{2}$ and smaller, we prepared electrode patches using CD-Rs. Impedance was measured against a well defined macroscopic (cm-dimension) iridium sputtered titanium electrode. As test electrolyte served biologically relevant PBS (phosphate buffered saline) - dilution series from $140 \mathrm{mM}$ down to $0.55 \mathrm{mM}$. A front end with high ohmic monitor amplifier [62;63] was used to interface the electrode to the gain-phase analyzer (Solartron 1260).

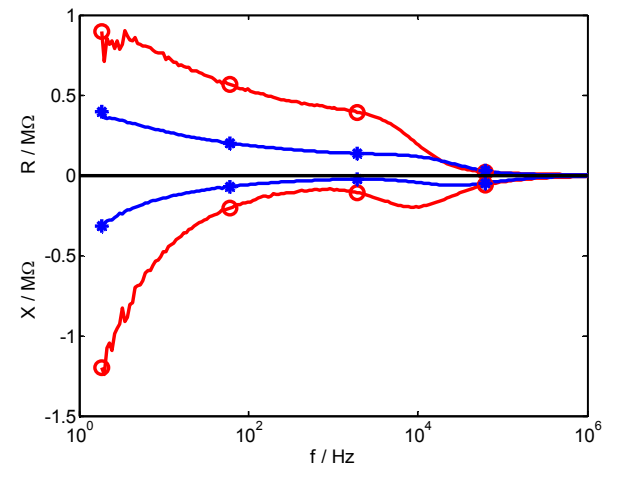

Fig.13: Impedance spectrum (real and imaginary part) of a $0.125 \mathrm{~mm}^{2}$ - gold electrode made from a CD-R. (o - $0.055 \mathrm{mM}$ PBS, * - $2.18 \mathrm{mM}$ PBS).

The identification of the confidence region for a single Aumicroelectrode with surface area below $1 \mathrm{~mm}^{2}$ is somehow arbitrary, since the impedance changes over the entire spectrum from $10 \mathrm{~Hz}$ up to $1 \mathrm{MHz}$. 


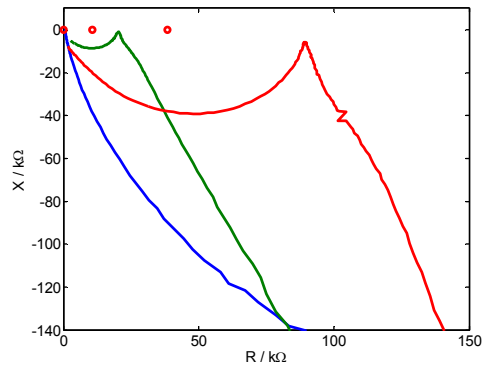

b
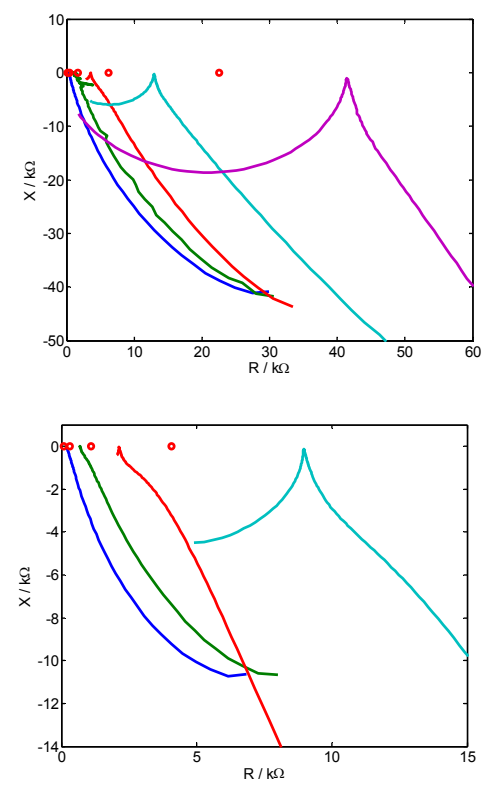

Fig.14: Impedance of gold electrodes in PBS of different concentration and different surface area: a) $A=1 \mathrm{~mm}^{2}$, concentration from left to right: $140 \mathrm{mM}, 2.18 \mathrm{mM} 0.55 \mathrm{mM} \mathrm{b})$ $\mathrm{A}=4 \mathrm{~mm}^{2}, \mathrm{c}=140 \mathrm{mM}, 35 \mathrm{mM}, 8.75 \mathrm{mM}, 2.18 \mathrm{mM}, 0.55 \mathrm{mM}$ and c) $\mathrm{A}=16 \mathrm{~mm}^{2}, \mathrm{c}=140 \mathrm{mM}, 35 \mathrm{mM}, 8.75 \mathrm{mM}, 2.18 \mathrm{mM}$. The red circles show the resistance estimated from the conductivity of the electrolyte and the geometry of the chamber inclusively the spreading resistance in series with the charge transfer resistance got from cyclic voltammetry. The resistance of the counter electrode was taken into account as well but it was small with only few percent of the charge transfer resistance.

In good approximation we used $1 \mathrm{kHz}$ as frequency where the impedance is mostly governed by the impedance of the electrolyte. The confidence interval increased with increasing electrode area and extends from $100 \mathrm{~Hz}$ up to $200 \mathrm{kHz}$ for the $16 \mathrm{~mm}^{2}$ electrode. For further comparison of the larger electrodes their Wessel diagram for different dilution of PBS is shown in Fig.14. By fast eye inspection it seems that the behaviour shown theoretically in Fig.2 is found in our measurements.

However, using the resistance of the saline, the spreading resistance as well as the charge transfer resistance, derived from the slope of the CV-diagram (Fig.15.), it was impossible to account quantitatively for the electrode impedance as presented in Fig.2.

The measurements were done with a stimulating voltage of $100 \mathrm{mV}_{\mathrm{pp}}$, e.g. the actual voltage ranged from $-50 \mathrm{mV}<U<50 \mathrm{mV}$. Thus, for assessing the charge transfer resistance the slope of the $U / I$ - characteristic in Fig.15a is relevant. Of course, just looking for the current at a given voltage should work theoretically, but with the data in Fig. 15a, it is clear that the slope between $-50 \mathrm{mV}$ and $50 \mathrm{mV}$ would be more reliable. Note that the slope $\Delta I / \Delta U$ is the small signal conductance and should be in good approximation the reciprocal of the charge transfer resistance $R_{c}$. The current offset is not necessarily due to an offset in the instrument but appears at metal electrodes in general, especially when corrosion takes place. It should be noted that Fig. 15 shows the measurement for 140 mM PBS.
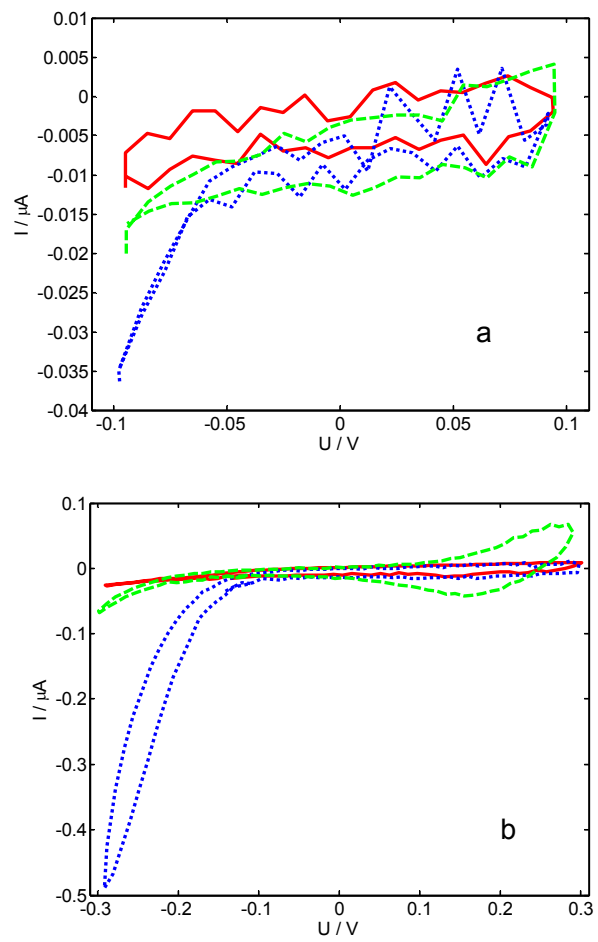

Fig.15: Cyclic voltammogram (CV) for gold electrodes of different area in $140 \mathrm{mM}$ PBS (solid: $1 \mathrm{~mm}^{2}$, dotted: $4 \mathrm{~mm}^{2}$, dashed: $16 \mathrm{~mm}^{2}$ ), a) $-100 \mathrm{mV}<U<100 \mathrm{mV}$, b) $-300 \mathrm{mV}<U<300 \mathrm{mV}$. The ramp steepness was set to 10 $\mathrm{mV} / \mathrm{s}$. An $\mathrm{Ag} / \mathrm{AgCl}$ - electrode served as reference while a massive platinum electrode was used as counter electrode.

The slope for lower concentration is even smaller with more noise, making the assessment of the charge transfer resistance increasingly questionable with decreasing conductivity of the electrolyte. In our experiments, it reached even several M $\Omega$. As seen in Fig.13 and 14, the maximum resistance found was much less than $\mathrm{M} \Omega$ at $1 \mathrm{kHz}$ even for the smallest electrode contacting the less conductive electrolyte which means that the charge transfer resistance is already shortened by the resistance $X_{c}$ of the double layer capacitance. Assuming an average area specific capacity of the electric double of $C_{d l}=30 \mu \mathrm{Fcm}^{-2}$, which gives a capacitive resistance at $1 \mathrm{kHz}$ for the $1 \mathrm{~mm}^{2}$ electrode of

$$
X_{C}=\frac{1}{2 \pi f C_{d l} A_{d l}}=\frac{1}{2 \pi \cdot 1 \mathrm{kHz} \cdot 30 \mu \mathrm{Fcm}^{-2} \cdot 1 \mathrm{~mm}^{2}}=530 \Omega
$$

which is far less than the total resistance measured at $1 \mathrm{kHz}$, where the Warburg impedance becomes negligible. 
This excludes the interpretation that the half circle visible is due to the impedance of the double layer. However, as seen for the lowest electrolytic concentration in Fig.14c, the combination of Warburg and Nernst-impedance as shown in Fig.2 is evident as well but with only a small rest of the half circle left before the curve reaches the x-axis. Closer examination shows, that the feature shown in Fig.2 works well with big electrodes, such as parallel plates but vanishes with smaller electrode dimension.

The point, where the real axis ( $\mathrm{x}$-axis) is reached should be the impedance of the electrolyte. This can be calculated because of the simple geometry of the chamber and was found too small by almost an order of magnitude. Even the impedance of the counter electrode in series, which is only $1-2 \%$ of the total impedance at $1 \mathrm{kHz}$ will not considerably change the situation.

Something very important is the spreading resistance. Taking a very small electrode in a big chamber will concentrate the electric field lines near to the electrode, thereby creating a high resistance in this region as one can estimate it from the electric field distribution simulated in Fig.16.

Using a simple approximation, the spreading resistance extends from the edge of a disk shaped electrode to the edge of the chamber (Fig.17).

The onion like layers of differential spreading resistance $d R_{s}$ are assumed to be spherical and increase their area with the distance $\mathrm{x}$ from the electrode.

$$
d R_{s}=\frac{d x}{2 \pi \kappa x^{2}}
$$

Integrating from the electrode radius, $r_{e l}$, to the radius of the chamber, $r_{c h}$, yields

$$
R_{s}=\frac{d x}{2 \pi \kappa x^{2}} \int_{r_{e l}}^{r_{c h}} \frac{d x}{x^{2}}=\frac{1}{2 \pi \kappa}\left(\frac{1}{r_{e l}}-\frac{1}{r_{c h}}\right)
$$

The importance of the spreading resistance should be considered with respect to the dimension of the chamber. One limiting case is $r_{e l}=r_{c h}$ where $R_{s}$ becomes zero. This is the usual case for parallel plate electrodes. The other case is $r_{e l}$ $<<r_{c h}$ where $r_{c h}$ can be considered infinite, i.e. an electrode with negligible dimension resides in a big chamber (pointelectrode) which results in

$$
R_{s}=\frac{1}{2 \pi \kappa r_{e l}} .
$$

In conclusion, the current in the vicinity of a point electrode assesses an area which increases with the square of the radius resulting in a decrease of the current density. This effect vanishes with increasing electrode surface with respect to the dimension of the chamber.

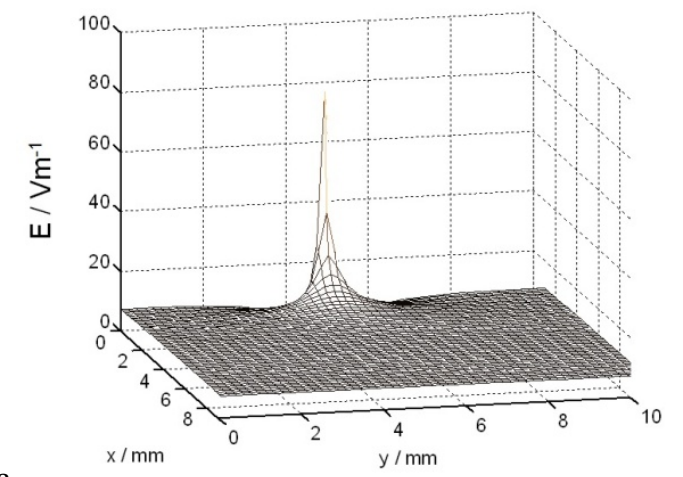

$\mathrm{a}$

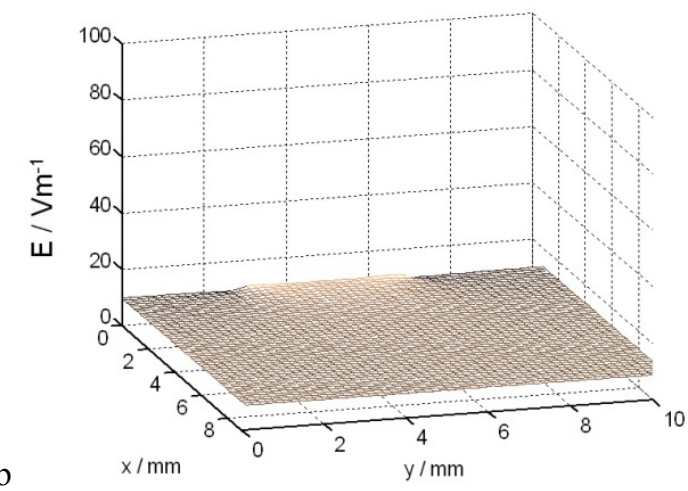

Fig.16: Electric field strength near the electrode: a) $\emptyset_{\text {electrode }}=0.2 \mathrm{~mm}$ and b) $\emptyset_{\text {electrode }}=4 \mathrm{~mm}$ in a cylindric chamber with a diameter of $10 \mathrm{~mm}$ with a voltage of $100 \mathrm{mV}$ applied against a distant electrode at $10 \mathrm{~mm}$. Only the plane in the middle of the chamber is shown.

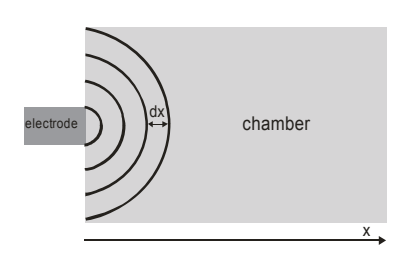

Fig.17: Simple approach for calculating the spreading resistance of a small disk shaped electrode

Taking into account the distribution of the resistance along the x-axis (Fig.17) when doing electrical measurements means that most information about the MUT is gained from the immediate vicinity of the electrode. On one hand, this is wanted for confinement of the measurement, e.g. at the tip of an AFM-probe, but is a real problem if small electrodes are used for monitoring the behaviour of big objects.

Even by taking the spreading resistance into account, the data in Fig.14 could not be well fitted. The circles along the $x$-axis correspond to the sum of $R_{s}, R_{\text {counterelectrode, and }}$ $\mathrm{R}_{\text {saline. }}$. Finally, the measured resistance is in all cases too high.

The origin of the semicircle extending to $R=0 \Omega$ is the parasitic capacitance of the measuring chamber filled with electrolyte. It is not, however, just the intrinsic relaxation of the electrolyte with the characteristic frequency $f_{0}$ which is even for the lowest conductivity used here $(\kappa=0.087$ $\left.\mathrm{mScm}^{-1}\right)$ and the relative permittivity of water $\left(\varepsilon_{\mathrm{r}}=78\right)$, 
$\mathrm{f}_{0, \text { electrolyte }}=\kappa / 2 \pi \varepsilon_{\mathrm{r}} \varepsilon_{0}$ still more than $2 \mathrm{MHz}$ which is by far more than measured $(100 \mathrm{kHz}-1 \mathrm{MHz})$.

This however, does not mean that proper calibration of these electrodes was not possible. As seen in Fig.18, a linear relationship between the measured conductance and the known (independently measured) conductivity of the MUT exist for all the electrode geometries.

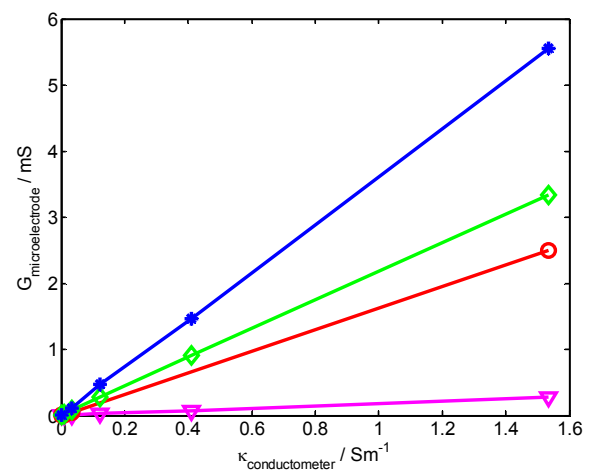

Fig.18: Comparison between conductivity measured with a conductometer (LF-300, WTW Weinheim) and by impedance measurement using gold electrodes of different size $\left(\Delta 0.12 \mathrm{~mm}^{2}\right.$, o $1 \mathrm{~mm}^{2}, \diamond 4 \mathrm{~mm}^{2}$, and $* 16 \mathrm{~mm}^{2}$ ).

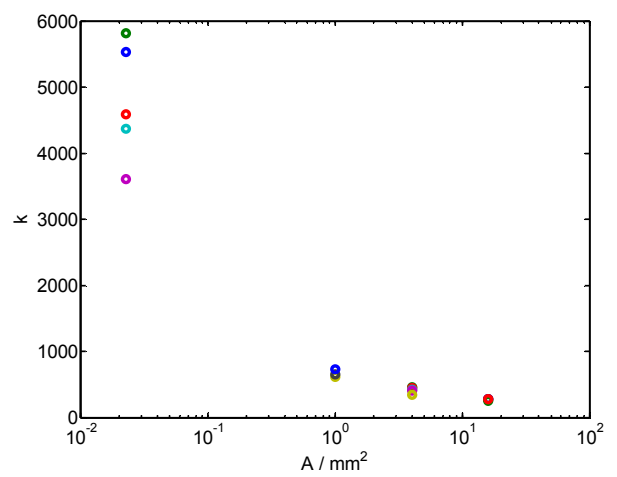

Fig. 19: Mean value of the geometry factor $k(1 \mathrm{kHz}-10 \mathrm{kHz})$ for gold electrodes with different surfaces area. This different behavior for the very small electrode suggests an increasing influence of parasitic elements. Compensating for parasitic elements requires that they are independent on the experimental condition and that a suitable model is used which needs to be tested individually for all electrode geometries.

Calculating the geometry factor for the bigger electrodes yields sufficiently reproducible results (Fig.19). But with decreasing electrode size a systematic error becomes noticeable. As seen in Fig.19, $k$ spreads from almost 6000 for the highest conductivity of the electrolyte $(140 \mathrm{mM}$ PBS, $15.35 \mathrm{mS} / \mathrm{cm})$ down to 3500 for the lowest one $(0.0869 \mathrm{mS} / \mathrm{cm})$. Using a mean value for the geometry factor for calibration of the electrodes, Kohlrausch's law can be tested as well (Fig.20).

Even though Kohlrausch's law works over a wide range for macroscopic electrodes, like for instance electrode systems as shown in Fig.6, an increasing scatter in the data prevents accurate measurements when the electrode area is below about $0.2 \mathrm{~mm}^{2}$. Because Kohlrausch's law is very sensitive to data scatter, it can serve for quality assessment of miniaturized electrodes.

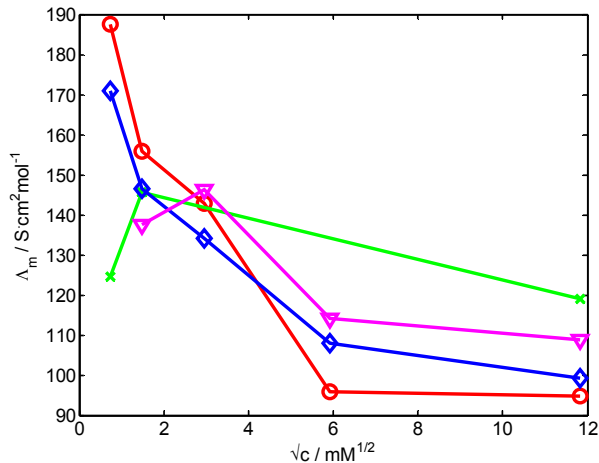

Fig.20: Molar conductivity vs. square root of the concentration for gold electrodes of different size $\left(\diamond 0.125 \mathrm{~mm}^{2}, \mathrm{x} 1 \mathrm{~mm}^{2}\right.$, o 4 $\mathrm{mm}^{2}, \Delta 16 \mathrm{~mm}^{2}$ )

\section{Non-linearity of electrode behaviour}

If the exciting voltage is not more than $100 \mathrm{mV}$, one would not expect any non-linearities in the measurement associated with the electrodes. However, when minimizing electrodes, high curvature appears. As shown in Fig.16, this concentrates the field so that almost all voltage can drop in these regions. According to Fig. 15 even $100 \mathrm{mV}$ can already drive an electrode system into a non linear region of the U/I-characteristic.

\section{Galvanically decoupled electrodes}

Electrode systems passivated by a dielectric layer show an almost pure capacitive behavior. The capcitance of this layer is important for processing the data since this impedance is in series to the MUT. It is not always simple to measure the thickness by AFM or ellipsometry. Such methods fail when electrode surfaces are rough. For electrodes shown in Fig. 8 we used the empty chamber and compared the measured capacity with this measured with the chamber filled with mercury. Since the capacity of the dielectric passivation layer is not changing, for instance by taking up water, it can be used to correct the data measured with other conductive MUT. An example of calibration result for the $20 \mu \mathrm{m}$ - electrode (Fig.8) is given in Fig 21 . A clear functional dependence between the electrolyte conductivity and the impedance measured by this microelectrode was found for higher frequency ( $>10 \mathrm{kHz}$ ) and for low conductivity of the MUT (Fig.22). The sensitivity of the electrode system vanishes with higher concentration. Below about $10 \mathrm{kHz}$ only capacitive behavior with an impedance locus going staight down the $\mathrm{y}$-axis was found.

Despite the functional dependence between the impedance at higher frequency and the impedance of the electrode, quantitative analysis fails up to date. One reason is the instability of the measuring chamber due to the use of materials with a high water uptake and thus sometimes noticable changes of the parasitic elements of the chamber. 


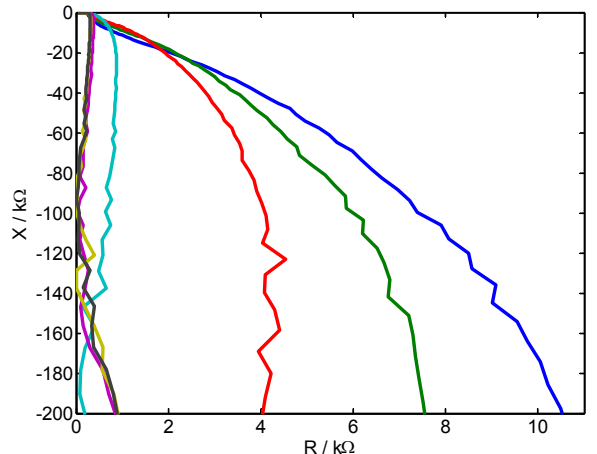

Fig.21: Locus diagram for the impedance of a $3 \mu \mathrm{m}$ interdigitated electrode immersed in $\mathrm{KCl}$-solution of different concentration. (along the arrow: $1 \mu \mathrm{M}, 10 \mu \mathrm{M}, 100 \mu \mathrm{M}, 1 \mathrm{mM}, 10 \mathrm{mM}, 100$ $\mathrm{mM}, 1 \mathrm{M}$ ). The frequency ranged from $500 \mathrm{~Hz}$ to $40 \mathrm{MHz}$. Note the different scaling of the axes.

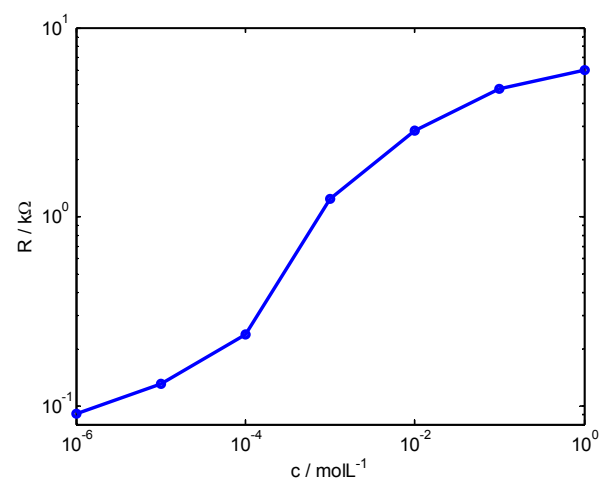

Fig.22: Functional dependence of the measured real part of the impedance at $10 \mathrm{kHz}$ with respect to the concentration of the $\mathrm{KCl}-$ solution

\section{Discrimination of water and oil}

The use of aqueous droplets separated by paraffine oil gives the opportunity to grow cells separately in miniaturized volumina sequentially arranged in a tube [64]. Sensors in these systems detect the appearance of these droplets, for instance for synchonizing subsequent sensors, e.g. for viability test. Since the surface inside the microfluidic device is important, any gavanically coupled electrode may be harmful for the system. The impedance difference found with an empirical approach, where a galvanically decoupled micro electrode system is mounted in a fluid chamber, is shown in Fig.23.

Despite the changes in impedance between both materials are very small, they are reproducible making this approach useful. However, quantitative analysis, like life/dead test of cells or counting of cells is not possible with these sensors. It is always advantageous to measure over a wide frequency range and to use the ratio of impedance magnitude at high and low frequency. This is especially useful if the impedance changes only in a part of the spectrum but stays indifferent for instance at high frequency.
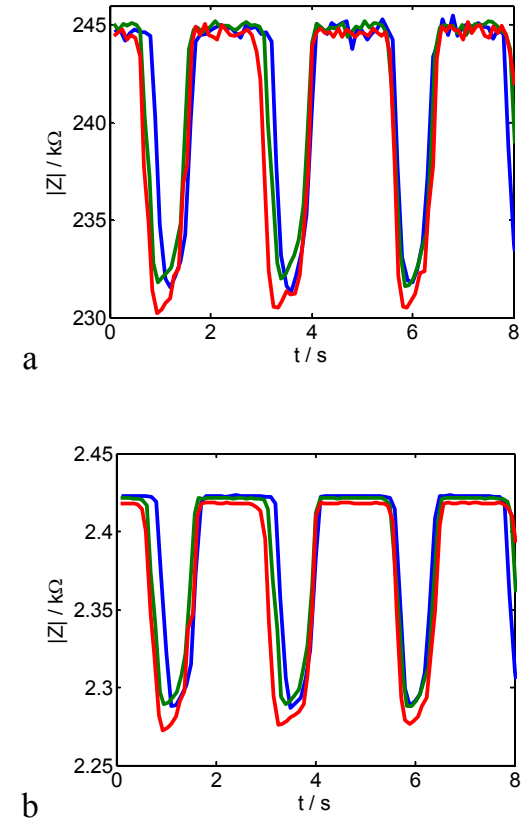

Fig.23: Change in impedance magnitude at $46 \mathrm{kHz}$ (a) and 4.5 $\mathrm{MHz}(\mathrm{b})$ of a segmented flow of paraffine oil and $\mathrm{KCl}$ - solution in a $0.5 \mathrm{~mm}$ tube. The flow was set to about 2 droplets per second.

The advantage of the electric discrimination of droplets is the very simple and robust setup compared for instance to optical measurements.

\section{Conclusion}

Despite microelectrodes can be made in a great variety and with almost any geometry, they become most useful when quantitative measurements reliably with a high sensitivity are possible.

Bipolar interfaces show several advantages like a simpler construction but also a higher robustness during the measurement, because only two electrodes need to be covered by the MUT. Especially, if the MUT reaches the dimension of the electrode (e.g. single cells), a proper placement of the MUT sometimes fails. The disadvantage of bipolar electrodes, the electrode impedance in series to the MUT can be compensated using subsequent measurements with the electrode system filled with electrolyte only and then with the test system like cells. This however, works only properly, if the electrode impedance will not change. This can be assumed, if a galvanostatic setup is used. If the excitation current source is offset free and symmetric with respect to ground, the electrode impedance will stay constant and can be easily compensated. If it is economically feasible, the electrode should have fractal surface, thereby minimizing the current density and thus the electrode polarization. Since this reduces the electrode impedance even up to magnitudes, the measurement noise reduces considerably.

Tetrapolar electrodes should be used when they are applicable, even when miniaturized electrodes are required. Despite it seems that the impedance of the current carrying 
electrodes is not important, it can greatly increase the noise of the measurement. Using potentiometric excitation, the voltage must be high enough in order to have a sufficient current flow through the electrodes which will drive most electrodes already into a non linear range of the current/voltage characteristic. Moreover, if nearly all voltage drops at the electrodes, only small variation of the electrode behavior may change the measurement result significantly. But even by using a galvanostatic approach, a high voltage drop at the electrode-electrolyte interface will drive the electrode into a non linear region.

The electrodes used for voltage monitoring are highly critical with respect to stability. If applicable, reversible electrodes, like $\mathrm{Ag} / \mathrm{AgCl}$-electrodes should be used. The dimension of these electrodes should be as small as possible, since they behave like floating electrodes carrying a current along the surface, even under ac-condition. Although only negligible currents are involved, during a half period of excitation at the positive side of the electrode takes an anodic reaction place while a cathodic reaction can be found at the opposite side. Even if the voltage is much below die dissociation voltage, the sensitive equilibrium between metal and metal ions will be disturbed. Moreover, if reversible electrodes are used, electrochemical reactions are proceeding even at very small current and voltage difference. Any electrochemical changes due to diffusion in the electric field will not fully reverse during the time of opposite voltage due to asymmetric transport in a high diffusion gradient.

The best monitor electrode, if bandwidth is unimportant, is an $\mathrm{Ag} / \mathrm{AgCl}$-electrode immersed in $3 \mathrm{M} \mathrm{KCl}$ and connected to the microscopic object with a micropipette as it is used in patch clamping.

The connection electronics (front end) should be symmetric with respect to ground in order to avoid asymmetric load to the electrodes, thereby causing unpredictable chemical reactions.

Even miniaturized electrodes fulfill Kohlrasch's law, however, the noise increases considerably when the electrode dimension decreases. Thus, even by designing microelectrodes, it is desirable to make them as big as possible for the particular application.

\section{References}

1. Yang L., Electrical impedance spectroscopy for detection of bacterial cells in suspensions using interdigitated microelectrodes., Talanta 2008;74(5):1621-9.

2. Zheng S, Liu M, Tai YC., Micro coulter counters with platinum black electroplated electrodes for human blood cell sensing., Biomed Microdevices 2008;10(2):221-31.

3. Asphahani F, Zhang M., Cellular impedance biosensors for drug screening and toxin detection., Analyst 2007; 132(9):835-41.
4. Jang LS, Wang MH., Microfluidic device for cell capture and impedance measurement., Biomed Microdevices 2007;9(5):737-43.

5. Zhou H, Tilton RD, White LR., The role of electrode impedance and electrode geometry in the design of microelectrode systems., J Coll Interf Sci 2006;297:819-31.

6. Pernkopf W, Sagl M, Fafilek G, Besenhard JO, Kronberger $\mathrm{H}$, Nauer GE., Applications of microelectrodes in impedance spectroscopy., Solid State Ionics 2005;176:20316.

7. Price DT, Rahman ARA, Bhansali S., Design rule for optimization of microelectrodes used in electric cellsubstrate impedance sensing (ECIS)., Biosensors and Bioelectronics 2008;24(7):2071-6.

8. Grimnes S, Martinsen OG. Bioimpedance and Bioelectricity Basics. Academic Press; 2000.

9. Foster KR, Schwan HP., Dielectric properties of tissues and biological materials : A critical review., CRC Crit Rev Biomed Eng 1989;17:25-104.

10. Muller MR, Salat A, Pulaki S, Stangl P, Ergun E, Schreiner $\mathrm{W}$, et al., Influence of hematocrit and platelet count on impedance and reactivity of whole blood for electrical aggregometry., J Pharmacol Toxicol Methods 1995;34(1):17-22.

11. Zhao TX, Lockner D., Electrical impedance and erythrocyte sedimentation rate (ESR) of blood., Biochim Biophys Acta 1993;1153(2):243-8.

12. Pethig R, Kell DB., The passive electrical properties of biological systems: their significance in physiology, biophysics and biotechnology., Phys Med Biol 1987;32(8):933-70.

13. Damez JL, Clerjon S, Abouelkaram S, Lepetit J., Beef meat electrical impedance spectroscopy and anisotropy sensing for non-invasive early assessment of meat ageing., J Food Engineering 2008;85(116):122.

14. Oliver MA, Gobantes I, Arnau J, Elvira J, Riu P, Grebol N, et al., Evaluation of the electrical impedance spectroscopy (EIS) equipment for ham meat quality selection., Meat Sci 2001;58:305-12.

15. Chanet M, Riviere C, Eynard P., Electric impedance spectrometry for the control of manufacturing process of comminuted meat products., J Food Engineering 1999;42:153-9.

16. Nacke T, Bruckner K, Goller A, Kaufhold S, Nakos X, Noack S, et al., New type of dry substances content meter using microwaves for application in biogas plant., Analytical Bioanalytical Chemistry 2006;383:252-7.

17. Kell DB, Markx GH, Davey CL, Todd RW., Real-time monitoring of cellular biomass. Methods and applications., Trends Anal Chem 1990;9:190-4.

18. Orazem ME, Tribollet B., An integrated approach to electrochemical impedance spectroscopy., Electrochimia Acta 2008;53(25):7360-6.

19. K'Owino IO, Sadik OA., Impedance Spectroscopy: A Powerful Tool for Rapid Biomolecular Screening and Cell Culture Monitoring., Electroanalysis 2005;17(23):2101-13. 
20. Sun T, Holmes D, Green NG, Morgan H., High speed multifrequency impedance analysis of single particles in a microfluidic cytometer using maximum length sequences., Lab Chip 2007;7:1034-40.

21. Watkins N, Venkatesan BM., A robust electrical microcytometer with 3-dimensional hydrofocusing., Lab on a Chip 2010;9(22):3177-84.

22. Ghanbari K, Bathaie SZ, Mousavi MF., Electrochemically fabricated polypyrrole nanofiber-modified electrode as a new electrochemical DNA biosensor., Biosens Bioelectron 2008;23(12):1825-31.

23. Varshney M, Li Y., Double interdigitated array microelectrode-based impedance biosensor for detection of viable Escherichia coli O157:H7 in growth medium., Talanta 2008;74(4):518-25.

24. Linderholm P, Marescot L, Loke MH, Renaud P., Cell culture imaging using microimpedance tomography., IEEE Trans Biomed Eng 2008;55(1):138-46.

25. Cantrell DR, Inayat S, Taflove A, Ruoff RS, Troy JB., Incorporation of the electrode-electrolyte interface into finite-element models of metal microelectrodes., J Neural Eng 2008;5(1):54-67.

26. Panescu D, Webster JG, Stratbucker RA., A nonlinear finite element model of the electrode-electrolyte- skin system., IEEE Trans Biomed Eng 1994;41(7):681-7.

27. Ahuja AK, Behrend MR, Whalen JJ, Humayun MS, Weiland JD., The Dependence of Spectral Impedance on Disc Microelectrode Radius., IEEE Trans Biomed Eng 2008;55(4):1457-60.

28. Schwan HP. Electrical Properties of Tissue and Cell Suspensions. In: Lawrence JH, Tobias CA, editors.New York: Academic Press; 1957.p. 147.

29. Schwan HP., Mechanisms responsible for electrical properties of tissues and cell suspensions., Med Prog Technol 1993;19(4):163-5.

30. Pauly H, Schwan HP., Uber die Impedanz einer Suspension von kugelformigen Teilchen mit einer Schale., Z Naturforsch 1957;14(b):125-31.

31. Raicu V, Raicu G, Turcu G., Dielectric properties of yeast cells as simulated by the two-shell model., BBA 1996;1274:143-8.

32. Feldmann Y, Ermolina I, Hayashi Y., Time Domain Spectroscopy Study on biological Systems., IEEE Transactions on Dielectrics and Electrical Insulation 2003;10:728-53.

33. Gabriel S, Lau RW, Gabriel C., The dielectric properties of biological tissues: III Parametric models for the dielectric spectrum of tissues., Phys Med Biol 1996;41:2271-93.

34. Angersbach A, Heinz V, Knorr D., Electrophysiological model of intact and processed plant tissues:cell disintegration criteria., Biotechnol Prog 1999;15:753-62.

35. Holmes D, Pettgrew D, Reccius CH, Gwyer JD, van Berkel $\mathrm{C}$, Holloway J, et al., Leukocyte analysis and differentiation using high speed microfluidic single cell impedance spectroscopy., Lab on a Chip 2009;9:2881-9.
36. Bragos R, Sarro E, Fontova A, Soley A, Cairo J, BayesGenis A, et al., Four versus two-electrode measurement strategies for cell growing and differentiation monitoring using electrical impedance spectroscopy., Conf Proc IEEE Eng Med Biol Soc 2006;1:2106-9.

37. Urdapilleta E, Bellotti M, Bonetto FJ., Impedance analysis of cultured cells: a mean-field electrical response model for electric cell-substrate impedance sensing technique., Phys Rev E Stat Nonlin Soft Matter Phys 2006;74(4 Pt 1):041908.

38. Fomekong RD, Pliquett U, Pliquett F., Passive electrical properties of RBC suspensions: changes due to distribution of relaxation times in dependence on the cell volume fraction and medium conductivity., Bioelectrochem and Bioenerg 1998;47:81-8.

39. Cha K, Brown EF, Wilmore DW., A new bioelectrical impedance method for measurement of the erythrocyte sedimentation rate., Physiol Meas 1994;15(4):499-508.

40. Lee SW, Tai YC., A micro cell lysis device., Sensors and Actuators 1999;73(1-2):74-9.

41. Suehiro J, Shutou M, Hatano T, Hara M., High sensitive detection of biological cell using dielectrophoretic impedance measurement method combined with electropermebilization., Sensors and Actuators 2003;96:14451.

42. Davey CL, Davey HM, Kell DB., Introduction to the Dielectric Estimation of Cellular Biomass in Real Time, with Special Emphasis on Measurements at high Volume Fractions., Analytica Chemica Acta 1993;279:155-61.

43. Agilent Technologies. Agilent Impedance Measurement Handbook: A guide to measurement and techniques. 2009. Ref Type: Data File

44. Nelson SO, Bartley Jr. PG., Frequency and temperature dependence of the dielectric properties of food materials., Transactions of the ASAE 2002;45(4):1223-7.

45. Mirtaheri P, Grimnes S, Martinsen OG., Electrode polarization impedance in weak $\mathrm{NaCl}$ aqueous solutions., IEEE Trans Biomed Eng 2005;52(12):2093-9.

46. Ragheb T, Geddes LA., The polarization impedance of common electrode metals operated at low current density., Ann Biomed Eng 1991;19(2):151-63.

47. Cole KS. Membranes, Ions and Impulses. University of California Press; 1968.

48. Gabriel S, Lau RW, Gabriel C., The dielectric properties of biological tissues: II. Measurements in the frequency range 10 Hz to 20 GHz., Phys Med Biol 1996;41:2251-69.

49. Hamann $\mathrm{CH}$, Vielstich W. Elektrochemie. Weinheim: Wiley-VCH Verlag GmbH; 1998.

50. Troy JB, Cantrell DR, Taflove A, Ruoff RS., Modeling the electrode-electrolyte interface for recording and stimulating electrodes., Conf Proc IEEE Eng Med Biol Soc 2006;1:87981.

51. Zhou H, Tilton RD, White LR., The role of electrode impedance and electrode geometry in the design of microelectrode systems., J Colloid Interface Sci 2006;297(2):819-31. 
52. Franks W, Schenker I, Schmutz P, Hierlemann A., Impedance characterization and modeling of electrodes for biomedical applications., IEEE Trans Biomed Eng 2005;52(7):1295-302.

53. Zhou H, Preston MA, Tilton RD, White LR., Calculation of the dynamic impedance of the double layer on a planar electrode by the theory of electrokinetics., J Coll Interf Sci 2005;292:277-89.

54. Koester O, Schuhmann W, Vogt H, Mokwa W., electrochemical impedance spectroscopy, Quality control of ultra-micro electrode arrays, using cyclic voltammetry and scanning electrochemical microscopy., Sensors and Actuators 2001;76:573-81.

55. Bates JB, Chu YT., Electrode-electrolyte interface impedance: experiments and model., Ann Biomed Eng 1992;20(3):349-62.

56. MacDonald JR. Impedance spectroscopy. New York: John Wiley \& Sons; 1987.

57. Ackmann JJ, Seits M.A., Methods of Complex Impedance Measurements in Biological Tissue., CRC Critical Revue in Biol Eng 1984;11(4):281-311.

58. Geddes LA., Who introduced the tetrapolar method for Measuring resistance and impedance?, IEEE Eingineering in Medicine and Biology 1996;133-4.

59. Paixao TR, Richter EM, Brito-Neto JG, Bertotti M., Fabrication of a new generator-collector electrochemical micro-device: Characterizations and applications., Electrochemistry Communications 2006;8:9-14.

60. Nacke T, Barthel A, Friedrich J, Helbig M, Sachs J, Peyerl $\mathrm{P}$, et al. A new hard and software concept for impedance spectroscopy analyzers for broadband process measurements. ICEBI 2007; Berlin-Heidelberg: Springer Verlag; 2007 p. 194-7.

61. Rahman ARA, Priece DT, Bhansali S., Effect of electrode geometry on the impedance evaluation of tissue and cell culture., Sensors and Actuators 2007;127:89-96.

62. McAdams ET, Lackermeier A, McLaughlin JA, Macken D, Jossinet J., The linear and non-linear electrical properties of the electrode-electrolyte interface., Biosensors and Bioelectronics 1995; 10:67-74.

63. McAdams ET, Jossinet J., The detection of the onset of electrode-electrolyte interface impedance nonlinearity: a theoretical study., IEEE Trans Biomed Eng 1994;41(5):498500 .

64. Grodrian A, Metze J, Henkel T, Martin K, Roth M, Koehler JM., Segment flow generation by chip reactors for highly parallelizid cell cultivation., Biosensors and Bioelectronics 2004;19(11):1421-8. 